\title{
Semianalytic Numerical Studies of Turning Points Arising in Stiff Boundary Value Problems
}

\author{
By W. L. Miranker* and J. P. Morreeuw
}

\begin{abstract}
A numerical algorithm for solving stiff boundary value problems with turning points is presented. The stiff systems are characterized as singularly perturbed differential equations. The numerical method is derived by appropriately discretizing the boundary layer and connection theory for such systems. Numerical results demonstrate the effectiveness of the method. In many cases the calculation proceeds with mesh increments which are orders of magnitude larger than those used by other known methods.
\end{abstract}

1. Introduction. Numerical methods for approximating the solution of boundary value problems subjected to singular perturbations have recently begun to appear (cf. F.W. Dorr [1] , A. M. Il'in [3] , R.E. O'Malley [5] , C. E. Pearson [6] , as well as unpublished work of $\mathrm{H}$. Keller and $\mathrm{H}$. Kress).

The canonical form of such a problem is

$$
\begin{gathered}
M y \equiv \epsilon y^{\prime \prime}+f(x) y^{\prime}+g(x) y=h(x), \quad 0<x<1, \\
y(0)=\alpha, \quad y(1)=\beta
\end{gathered}
$$

where $\epsilon$ is considered to be small.

Singularly perturbed problems arise commonly in applications so that numerical methods for approximating their solutions are of considerable interest. The interest is all the more enlarged when the observation is made that such problems comprise classes of problems of the so-called stiff type. Thus, numerical methods developed for singularly perturbed problems will automatically be of use for the numerical solution of stiff problems. This connection between these two classes of problems was noted already by one of us (cf. [4]) in a study concerning stiff initial value problems. In that study, as in the present one, the asymptotic methods, usually called boundary layer techniques, known to describe the solution of the singularly perturbed problem, are exploited to generate the numerical methods.

Received August 17, 1973.

AMS (MOS) subject classifications (1970). Primary 65L10, 34E20.

* This research was partially supported by ONR Contract N00014-69-C-0023. 
The boundary value problem (1.1) is further complicated enormously, when compared to the initial value problem, by the presence of points where $f$ vanishes, the so-called turning points. The analytic value of the solution in the presence of such points as well as the relationship between the values of the solution on adjoining sides of such points is the subject of the so-called connection theory (i.e., WKB analysis, cf. R. O'Malley [5]).

Our numerical methods deal with the turning points by casting the connection theory into a numerically exploitable form, moreover, in combination with the boundary layer methods just referred to.

The resulting numerical methods inherit the favorable feature of the analytic methods, namely, they improve rather than degrade with increasing stiffness (decreasing $\epsilon$ ) in the problem. Moreover, the mesh increments which are used are frequently orders of magnitude larger than those required by other numerical methods for similar problems.

In the present study, our results concern only the linear form (1.1) of the problem. We also set aside the especially difficult phenomena of resonance in the solutions which occurs when $f$ has multiple zeros or when at a simple zero of $f$ the quantity $g / f^{\prime}$ is an integer.

Our numerical method provides a pointwise approximation to the solution $y$ of (1.1) which is $O(\Delta x)$. The approximation is probably uniform within $O(\Delta x)$ in cases where the maximum principle prevails for (1.1). Improvements in the order of the approximation are directly obtainable, as may be seen.

In Section 2, we review the asymptotic theory of turning points which describes the structure of the solution of (1.1). We do this in a form which is designed for the numerical work to follow. In Section 3, we give the formal presentation of our algorithm consisting of a discretization of the boundary layer methods and connection theory developed in Section 2. In Section 4, we comment on limitations of the algorithm and we present an algebraic point of view which leads to a rapid iteration method for executing the algorithm introduced in Section 3. In Section 5, we give the results of calculations performed on a large number of cases.

2. Analytic Aspects of the Solution. In this section, we derive various analytic properties of solutions of (1.1).

2.1. Preliminaries. We proceed by introducing some terminology by means of the following definitions. In these definitions, all points and sets lie in $[0,1]$.

Definition 2.1. A point $x$ is said to be an irregular point if in every neighborhood of $x$, the function $f(x)$ is neither larger than a positive number nor smaller than a negative number.

Remark 2.1. Turning points are irregular points.

Definition 2.2. An interval of regularity is an interval containing no irregular points. 
Definition 2.3. A neighborhood of irregularity is an open interval containing exactly one irregular point.

Definition 2.4. A right (left) sided neighborhood of irregularity is an open interval containing no point of irregularity and whose greatest lower (least upper) bound is a point of irregularity. When we need not specify the right or left sidedness, we will refer to these neighborhoods as demineighborhoods of irregularity.

2.2. Form of the Solution in an Interval of Regularity. In a closed interval of regularity, the solution $y$ of (1.1) may be written in the form

$$
y=u+v
$$

with
(a) $v=e^{-\phi / \epsilon} w$,
(b) $\phi^{\prime}=f$,
(c) $\mathrm{Mu}=h$,
(d) $M v=0$.

From these, in turn, we obtain

$$
\epsilon w^{\prime \prime}-d(f w) / d x+g w=0 .
$$

Introducing the operators $L$ and its adjoint $L^{*}$ through

$$
\begin{aligned}
L z & \equiv f d z / d x+g z, \\
L^{*} z & \equiv-d(f z) / d x+g z,
\end{aligned}
$$

we may write (2.2) (c) and (2.2) (e), respectively, as
(a) $\epsilon u^{\prime \prime}+L u=h$,
(b) $\epsilon w^{\prime \prime}+L^{*} w=0$.

Indeed, (2.2) (e) may be written as

(c) $M^{*} w=0$,

where $M^{*}$ is the adjoint of $M$.

Remark 2.2. The decomposition (2.1) is unique up to $O(\epsilon)$ under suitable regularity conditions on $u, v, L u$ and $e^{-\phi / \epsilon} L^{*} w$.

To see this, let $y=u_{0}+v_{0}$ and $y=u_{1}+v_{1}$ be two such decompositions. Let $\hat{u}=u_{1}-u_{0}$ and $\hat{v}=v_{1}-v_{0}$ so that $\hat{u}+\hat{v}=0$. Then

$$
0=L(\hat{u}+\hat{v})=L \hat{u}+\left(e^{-\phi / \epsilon} L^{*}\left(e^{\phi / \epsilon} \hat{v}\right)\right)-\left(2 g-f^{\prime}\right) \hat{v}-f^{2} \hat{v} / \epsilon .
$$

Solving (2.5) for $\hat{v}$ and using suitable regularity conditions as cited gives $\hat{v}=O(\epsilon)$. Using this in turn along with $\hat{u}+\hat{v}=0$, we can obtain the same result for $\hat{u}$ demonstrating the remark. 
2.3. Form of the Solution in a Neighborhood of Irregularity. We will now review the connection theory which characterizes the behavior of solutions of (1.1) in neighborhoods of irregularity. We will follow the development of R. O'Malley with modifications which will be of importance for the numerical considerations to follow.

We will limit ourselves to the case in which $f$ has nonvanishing right and left sided derivatives at the irregular point, hereafter denoted by $x_{*}$. In each demineighborhood of $x_{*}, f$ may be written as

$$
f(x)=a\left(x-x_{*}\right)\left[1+1 / 2 f^{\prime \prime}\left(x_{*}+\theta\left(x-x_{*}\right)\right)\left(x-x_{*}\right) / a\right],
$$

where here and hereafter

$$
a=f^{\prime}\left(x_{*}\right) \text {. }
$$

We introduce the new variable $\eta$ in place of $x$ through

$$
\eta=\eta(x)=\left[2 / a \int_{x_{*}}^{x} f(s) d s\right]^{1 / 2}
$$

where

$$
\left(x-x_{*}\right) \eta(x) \geqslant 0 .
$$

Note that $\eta\left(x_{*}\right)=0, \eta^{\prime}\left(x_{*}\right)=1$ and that $\eta^{\prime}>0$ in the demineighborhood. Thus, the change of variables is a valid one and, from (1.1), we obtain

$$
\epsilon y_{\eta \eta}+\left(a \eta-\epsilon \eta^{\prime \prime} /\left(\eta^{\prime}\right)^{2}\right) y_{\eta}+a^{2} \frac{\eta^{2}}{x^{2}} \frac{g(x)}{\left(f^{\prime}(x)\right)^{2}} y=\frac{h(x)}{\left(\eta^{\prime}\right)^{2}} \text {. }
$$

The solutions of (2.10) are characterized by the following Proposition.

Proposition. There exist functions $M(\eta, \epsilon), N(\eta, \epsilon), \widetilde{h}(\eta, \epsilon)$ and $\sigma(\epsilon)$, analytic in $\epsilon$ and continuously differentiable in $\eta$ such that

$$
y(\eta, \epsilon)=M(\eta, \epsilon) z+\epsilon N(\eta, \epsilon) z_{\eta}
$$

is a solution of (2.10) where $z$ is a solution of

$$
\epsilon z_{\eta \eta}+a \eta z_{\eta}+(b+\epsilon \sigma(\epsilon)) z=\tilde{h}(\eta, \epsilon) .
$$

Here and hereafter

$$
b=g\left(x_{*}\right) .
$$

Proof. With

(a) $\zeta(\eta)=-\eta^{\prime \prime} /\left(\eta^{\prime}\right)^{2}$

(b) $\theta(\eta)=\left(a^{2} \eta^{2} g(x)-x^{2}\left(f^{\prime}(x)\right)^{2} b\right) / \eta x^{2}\left(f^{\prime}(x)\right)^{2}$,

(2.10) may be written as

$$
\epsilon y_{\eta \eta}+(a \eta+\epsilon \zeta(\eta)) y_{\eta}+(b+y \theta(\eta)) y=h(x) /\left(\eta^{\prime}\right)^{2} .
$$

Inserting (2.11) into (2.15) and using (2.12), we get

$$
A z+\epsilon B z_{\eta}+C=0,
$$


where

(a) $A=\eta\left(a M_{\eta}+\theta M\right)+\epsilon\left(M_{\eta \eta}+\zeta M_{\eta}-\sigma M-(b+\epsilon \sigma)\left(2 N_{\eta}+\zeta N\right)\right)$,

(b) $B=2 M_{\eta}-(N a \eta)_{\eta}+\theta \eta N+\zeta(M-N a \eta)+\epsilon\left(N_{\eta \eta}+\zeta N_{\eta}-\sigma N\right)$,

(c) $C=M \tilde{h}-h+\epsilon\left(N_{\eta} \tilde{h}+\zeta N \tilde{h}+(N \tilde{h})_{\eta}\right)$.

Setting

$$
M=\sum_{i=0}^{\infty} \epsilon^{i} M_{i} \quad \text { and } \quad N=\sum_{i=0}^{\infty} \epsilon^{i} N_{i},
$$

then the terms of order zero in $\epsilon$ in (2.17)(a) and (2.17)(b) yield

$$
\begin{aligned}
& \text { (a) } a M_{0, \eta}+\theta M_{0}=0 \\
& \text { (b) } 2 M_{0, \eta}-\left(N_{0} a \eta\right)_{\eta}+\theta \eta N_{0}+\zeta\left(M_{0}-a N_{0} \eta\right)=0
\end{aligned}
$$

Let $M_{0}$ be the solution of $(2.19)\left(\right.$ a) satisfying $M_{0}(0)=1$. Then the solution of (2.19)(b), which is bounded at $\eta=0$, is given by

$$
a N_{0} \eta=M_{0}-\exp \int_{0}^{\eta}(\theta / a-\zeta) d \eta
$$

Similarly, to order $i$ in $\epsilon$, the Eqs. (2.17) yield

$$
\begin{aligned}
& \text { (a) } \eta\left(a M_{i, \eta}+\theta M_{i}\right)-\sigma_{i-1} M_{0}+K_{i-1}(\eta)=0 \\
& \text { (b) }-\left(N_{i} a \eta\right)^{\prime}+(\theta / a) a \eta N_{i}-\zeta a \eta N_{i}+2 M_{i, \eta}+\zeta M_{i}+J_{i-1}=0 .
\end{aligned}
$$

Here, $K_{i-1}$ which depends on $M_{0}, \cdots, M_{i-1}, N_{0}, \cdots, N_{i-1}, \sigma_{0}, \cdots, \sigma_{i-2}$ is continuous at $\eta=0 . J_{i-1}$ depends on $N_{0}, \cdots, N_{i-1}, \sigma_{0}, \cdots, \sigma_{i-2}$.

Upon choosing $\sigma_{i-1}=K_{i-1}(0)$, (2.21)(a) may be solved for $M_{i}$ which is continuous at $\eta=0$. With this $M_{i},(2.21)(\mathrm{b})$ may be solved in turn for $N_{i}$ an with $N_{i}$ being bounded at $\eta=0$. In this manner, $M$ and $N$ may be constructed. Similarly, $\tilde{h}=\Sigma_{i=0}^{\infty} \tilde{h}_{i} \epsilon^{i}$ may also be obtained.

This demonstrates the Proposition.

If $z$ is a solution of (2.12) such that $\epsilon z_{\eta}$ is bounded, then from (2.11)

$$
y=M_{0} z+\epsilon N_{0} z_{\eta}+O(\epsilon) .
$$

Differentiating (2.11) with respect to $\eta$ and using (2.12) and the boundedness of $\epsilon z_{\eta}$ gives

$$
y_{\eta}=\left(M_{0}-N_{0} a \eta\right) z_{\eta}+O(1)
$$

From (2.22) it is then clear that if we restrict our attention to quantities determined up to $O(\epsilon)$, then we may use $z$, obtained from the restriction of (2.12), to

$$
\epsilon z_{\eta \eta}+a \eta z_{\eta}+b z=\tilde{h}_{0}(\eta)
$$

This equation possesses a solution of the form, $\tilde{h}_{0}(0) / b+O(\eta)+O(\epsilon)$, whose derivative, moreover is bounded with respect both to $\eta$ and to $\epsilon$. Thus, all bounded 
solutions $y$ of our original Eq. (1.1) may be written in the form

(a) $y=M_{0}\left(z+h\left(x_{*}\right) / g\left(x_{*}\right)\right)+\epsilon N_{0} z_{\eta}+O(\epsilon, \eta)$, $^{* *}$

(b) $y_{\eta}=\left(M_{0}-N_{0} a \eta\right) z_{\eta}+O(1)_{(\epsilon, \eta)}$,

where here $z$ is a bounded solution of the homogeneous equation (2.23), viz.

$$
\epsilon z_{\eta \eta}+a \eta z_{\eta}+b z=0 .
$$

For the bounded solution, it may be verified that $\eta z_{\eta}$ as well as $\epsilon z_{\eta \eta}$ are bounded. Thus, since $x-\eta=O\left(\eta^{2}\right)$, we find that $z(x)-z(\eta)=O(\eta)$ and $\epsilon z_{\eta}(x)-\epsilon z_{\eta}(\eta)=$ $O(\eta)$. Using these observations and the regularity of $M_{0}$ and $N_{0}$, we may write (2.24) as

$$
\begin{aligned}
& \text { (a) } y(x)=M_{0}\left(z+h\left(x_{*}\right) / g\left(x_{*}\right)\right)+\epsilon N_{0} z_{x}+O\left(\epsilon, x-x_{*}\right) \text {, } \\
& \text { (b) } y^{\prime}(x)=\left(M_{0}-N_{0} a\left(x-x_{*}\right)\right) z_{x}+O(1)_{(\epsilon, x)} .
\end{aligned}
$$

As we will see further on, we may adopt the normalization $M_{0}\left(x_{*}\right)=1$. Thus, (2.26) may be further simplified to

$$
\begin{aligned}
& \text { (a) } y=z+h\left(x_{*}\right) / g\left(x_{*}\right)+\epsilon N_{0}(0) z^{\prime}+O\left(\epsilon, x-x_{*}\right) \\
& \text { (b) } y^{\prime}=z^{\prime}+O(1)_{\left(\epsilon, x-x_{*}\right)}
\end{aligned}
$$

where $z$ is a solution of

$$
\epsilon z^{\prime \prime}+a\left(x-x_{*}\right) z^{\prime}+b z=0 .
$$

We are now directed to the solution of Eq. (2.28).

This equation has the parabolic cylinder functions for its solutions. We will now summarize the properties of these solutions which we will require for our numerical method.

In the following Table 2.1, $u_{1}$ and $u_{2}$ are approximations to independent solutions of (2.28) (which are bounded in neighborhoods of $x_{*}$ ). Here and throughout $p=b / a$. From this table, we can deduce properties of $z+\epsilon N_{0}(0) z^{\prime}$ and $z^{\prime}$ needed for $y$ and $y^{\prime}$ in (2.27) by taking appropriate (and as yet unspecified) combinations of $u_{1}$ and $u_{2}$. We recombine the entries in Table 2.1 calling them $Y_{i}, i=1,2$ where

$$
\begin{array}{ll}
\text { (a) } Y_{i}=u_{i}+\epsilon N_{0}(0) u_{i}^{\prime}, & i=1,2, \\
\text { (b) } Y_{i}^{\prime}=u_{i}^{\prime}, & i=1,2 .
\end{array}
$$

These quantities are needed for determining $y$ (as in (2.27)). In terms of our original notation, the relevant data are then displayed in the following Table 2.2.

In Table 2.2, $w_{2}$ and $w_{1}$ are, respectively, normalized solutions of $L w=0$

$$
{ }^{* *} f=O(x, y) \Rightarrow f /(|x|+|y|)<\text { const for }(|y|+|y|)<\text { const. }
$$


and $L^{*} w=0$. The normalization is such that there exist constants $p_{i}$ for $i=1,2$ such that

$$
\lim _{x \rightarrow x_{*}} \frac{w_{2}}{\left|x-x_{*}\right|^{p_{2}}}=\lim _{x \rightarrow x_{*}} \frac{f w_{1}}{\left|x-x_{*}\right|^{p_{1}}}=1 .
$$

Thus, from (2.27), we see that in each demineighborhood of irregularity the solution of (1.1) is a combination of $Y_{1}$ and $Y_{2}$, viz.

\begin{tabular}{|l||c|c|c|}
\hline & $u_{1}$ & $u_{2}$ & Restrictions \\
\hline$u(x)$ & $\exp \left[-\frac{a}{\epsilon} \int_{x_{*}}^{x} \sigma d \sigma\right]\left|x-x_{*}\right|^{p-1}$ & $\left|x-x_{*}\right|^{-p}$ & $\frac{\left|x-x_{*}\right|}{\epsilon}>1$ \\
\hline$u^{\prime}(x)$ & $-\frac{a}{\epsilon} \exp \left[-\frac{a}{\epsilon} \int_{x_{*}}^{x} \sigma d \sigma\right] \operatorname{sig}\left(x-x_{*}\right)\left|x-x_{*}\right|^{p}$ & $-\operatorname{sig}\left(x-x_{*}\right) p\left|x-x_{*}\right|^{-p-1}$ & $\frac{\left|x-x_{*}\right|}{\epsilon}>1$ \\
\hline$\left.u_{*}\right)$ & $2^{(p-1) / 2} \frac{\Gamma(1 / 2)}{\Gamma(1-p / 2)} \operatorname{Re}\left(\left(\frac{a}{\epsilon}\right)^{(1-p) / 2}\right)$ & $2^{-p / 2} \operatorname{Re}\left(\left(-\frac{a}{\epsilon}\right)^{p / 2}\right) \frac{\Gamma(1 / 2)}{\Gamma((1+p) / 2)}$ & \\
\hline$u^{\prime}\left(x_{*}\right)$ & $\operatorname{sig}\left(x-x_{*}\right) 2^{p / 2-1} \frac{\Gamma(-1 / 2)}{\Gamma((1-p) / 2)} \operatorname{Re}\left(\left(\frac{a}{\epsilon}\right)^{(1-p) / 2}\right)$ & $\operatorname{sig}\left(x-x_{*}\right)^{-(p+1) / 2} \frac{\Gamma(-1 / 2)}{\Gamma(p / 2)} \operatorname{Re}\left(\left(\frac{-a}{\epsilon}\right)^{(p+1) / 2}\right)$ \\
\hline
\end{tabular}

TABLE 2.1. Properties of Solutions of (2.28)

\begin{tabular}{|c|c|c|c|}
\hline & $Y_{1}(x)$ & $Y_{2}(x)$ & Restrictions \\
\hline$Y(x)$ & $\exp \left[-\frac{1}{\epsilon} \int_{x_{*}}^{x} f(s) d s\right] w_{1}(x)$ & $w_{2}(x)$ & $+O\left(\epsilon /\left(x-x_{*}\right), x-x_{*}\right)$ \\
\hline$Y(0)$ & $\frac{\operatorname{sig}\left(x-x_{*}\right)}{2 \epsilon} \frac{\Gamma(1 / 2)}{\Gamma(1-p / 2)} \operatorname{Re}\left(\left(\frac{a}{2 \epsilon}\right)^{-(p+1) / 2}\right)$ & $\frac{\Gamma(1 / 2)}{\Gamma((1+p) / 2)} \operatorname{Re}\left(\left(\frac{-a}{2 \epsilon}\right)^{p / 2}\right)$ & \\
\hline$Y_{x}(x)$ & $-\frac{f(x)}{\epsilon} \exp \left[-\frac{1}{\epsilon} \int_{x_{*}}^{x} f(s) d s\right] w_{1}(x)$ & $-\frac{g(x)}{f(x)} w_{2}(x)$ & $+O(1)$ \\
\hline$Y_{x}(0)$ & $\frac{1}{2 \epsilon} \frac{\Gamma(-1 / 2)}{\Gamma((1-p) / 2)} \operatorname{Re}\left((a / 2 \epsilon)^{-p / 2}\right)$ & $\frac{\Gamma(-1 / 2)}{\Gamma(p / 2)} \operatorname{sig}\left(x-x_{*}\right) \operatorname{Re}((-a / 2$ & $(p+1) / 2)$ \\
\hline
\end{tabular}

TABLE 2.2 


$$
\begin{aligned}
& \text { (a) } y=\frac{h\left(x_{*}\right)}{g\left(x_{*}\right)}+\lambda Y_{2}+\mu Y_{1}+O\left(\epsilon /\left(x-x_{*}\right), x-x_{*}\right), \\
& \text { (b) } y^{\prime}=\mu Y_{1}+O(1)_{\left(\epsilon /\left(x-x_{*}\right), x-x_{*}\right)}
\end{aligned}
$$

Thus, we see that up to terms which are $O(1)_{\left(\epsilon, x-x_{*}\right)}, Y_{1}$ may be identified with the function $v$ and $Y_{2}$ with the function $u$ introduced in (2.1). Thus, in an appropriate sense, the values of $u$ and $v$ and their derivatives at an irregular point may be read off from Table 2.2 .

This concludes our description of the solution of (1.1) in a neighborhood of irregularity.

3. Description of the Algorithm. In this section we derive our numerical method.

3.1. Preliminaries. Our calculation will be performed on the mesh of points, $\left\{x_{i} \mid i=0, \cdots, N\right\}$ where

$$
0=x_{0}<x_{1}<\cdots<x_{N}=1 .
$$

The mesh points consist of three types: irregular points, neighboring points and regular points. These are defined as follows:

Definition 3.1. An irregular mesh point is an irregular point in the sense of Definition 2.1. We assume that all irregular points are to be found among the mesh points. We also assume that each pair of irregular mesh points are separated by at least two points of the mesh.

Definition 3.2. $x_{i}$ is a neighboring mesh point if either $x_{i+1}$ or $x_{i-1}$ are irregular points.

Definition 3.3. $x_{i}$ is a regular mesh point if it is neither an irregular mesh point nor a neighboring mesh point.

We will hereafter drop the qualifying word, mesh, associated with these points, since no confusion will result.

Let $\hat{f}$ denote a discretization of $f$. That is a function which interpolates $f$ on the mesh. Similarly, $\hat{u}$ and $\hat{v}$ will denote discretizations of $u$ and $v$ respectively. $\hat{\phi}$ will denote a primitive of $\hat{f}$.

In addition to the usual forward and backward divided difference operators, which will be denoted by a subscript $x$ or $\bar{x}$ respectively, we will make use of a directional divided difference operator used by F. W. Dorr [1]. This is given by

$$
\widehat{a \frac{\partial}{\partial x}}(\cdot)= \begin{cases}a(\cdot)_{x}, & \text { if } a>0 \\ a(\cdot)_{\bar{x}}, & \text { if } a \leqslant 0\end{cases}
$$

The dual of this operator is given by

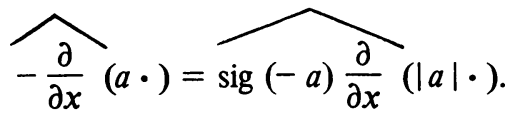


3.2. The Discretization at a Regular Point. At a regular point $x_{i}, M u=h$ and $M v=0$ are discretized as

$$
\epsilon(\hat{u})_{x \bar{x}, i}+\widehat{f_{i} \frac{\partial}{\partial x}}(\hat{u})_{i}+g_{i} \hat{u}_{i}=h_{i}
$$

and

$$
e^{-\hat{\varphi}_{i} / \epsilon}\left[\epsilon\left(\hat{v} e^{\hat{\varphi} / \epsilon}\right)_{x \bar{x}, i}-\frac{\hat{\partial}}{\partial x}\left(f \hat{v} e^{\hat{\varphi} / \epsilon}\right)_{i}+g_{i}\left(\hat{v} e^{\hat{\varphi} / \epsilon}\right)_{i}\right]=0
$$

respectively.

Since (3.5) may be multiplied by a constant, the choice of the primitive $\hat{\varphi}$ occurring therein is arbitrary.

Remark 3.1. The directionally discretized terms in (3.4) and (3.5) respectively involve $u_{i}$ and $u_{i+1}$ and $v_{i}$ and $v_{i-1}$ or they involve $u_{i}$ and $u_{i-1}$ and $v_{i}$ and $v_{i+1}$ depending on the sign of $f$.

3.3. Treatment at a Neighboring Point. The principal difficulty at a neighboring point $x_{i}$ involves the evaluation of the second divided difference. We are ill advised to use (3.4) and (3.5) at a neighboring point since the terms $(\cdot)_{x \bar{x}, i}$ will involve values at both an irregular point and at a neighboring point, these points delimiting a region of rapid change of $u$ and/or $v$, when $\epsilon$ is small. Let $x_{i}$ denote the irregular point for which $x_{i}$ is a neighbor.

We proceed to obtain an alternate approximation to the second derivatives. Let $r$ denote the second derivative of $u$. Then $(2.2)(\mathrm{c})$ becomes

$$
\epsilon r+f u^{\prime}+g u=h .
$$

Differentiating (3.6) gives

$$
f r+\left(f^{\prime}+g\right) u^{\prime}+g^{\prime} u=h^{\prime}-\epsilon r^{\prime} .
$$

Combining (3.6) and (3.7) gives

$$
r\left[f^{2}-\epsilon\left(f^{\prime}+g\right)\right]=(g u-h)\left(f^{\prime}+g\right)+f\left[\left(h^{\prime}-\epsilon r^{\prime}\right)+g^{\prime} u\right] .
$$

If the last bracket here is bounded at $x_{i}$, we may neglect it, since its coefficient $f$ is small at $x_{i}$; (provided of course that $\left|x_{i}-x_{i^{*}}\right|$ is small, which we assume). Then for $r$, we have the approximation

$$
\epsilon r=\chi_{1}(g u-h)
$$

where

$$
\chi_{1}=\epsilon\left(g+f^{\prime}\right) /\left(f^{2}-\epsilon\left(f^{\prime}+g\right)\right) .
$$

Similarly, denoting the second derivative of $w=e^{\hat{\varphi} / \epsilon} v$ by $s$, we obtain from (2.2)(d) the following four equations in place of (3.6), (3.7), (3.9) and (3.10), respectively,

$$
\epsilon s-f w^{\prime}+\left(g-f^{\prime}\right) w=0
$$




$$
-f s+\left(g-2 f^{\prime}\right) w^{\prime}+\left(g-f^{\prime}\right)^{\prime} w=-\epsilon s^{\prime}
$$

and

$$
\epsilon s=\chi_{2}\left(g-f^{\prime}\right) w
$$

where

$$
\chi_{2}=\epsilon\left(g-2 f^{\prime}\right) /\left(f^{2}-\epsilon\left(g-2 f^{\prime}\right)\right)
$$

Using $r$ and $s$ in (3.4) and (3.5), respectively, in place of the divided second differences, Remark 3.1 shows that one of the resulting equations does not make use of data at the irregular point. We use this equation to calculate the associated function $(u$ or $w)$ as the case may be at the neighboring point. We call this function the principal function (relative to this neighboring point). The remaining function is called the minor function.

Let $y$ denote the principal function ( $u$ or $e^{\left(\varphi-\varphi_{i}^{*}\right) / \epsilon} v$, as the case may be) and let $\bar{y}$ denote the minor function.

Consider first the case where $y$ is $u$. Then write (3.6) as

$$
L y=h-\epsilon r .
$$

We may solve this equation to obtain

$$
y=\lambda+\mu w,
$$

where $\lambda$ is a particular solution to (3.15), $\mu$ a constant and $w$ is a normalized solution (in the sense of (2.30)) of $L y=0$. Writing

$$
\hat{y}_{i}=\lambda+\mu \hat{w}_{i} \text {, }
$$

we may approximate $\lambda$ as

$$
\lambda=\left(h_{i}-\epsilon r_{i}\right) / g_{i},
$$

while

$$
\hat{w}_{i}=\left|x_{i}-x_{i^{*}}\right|^{-g_{i} / f_{i}^{\prime}}
$$

In (3.18), $r$ is computed by means of (3.9) and (3.10) and in (3.9) we may set $u$ equal to $\hat{y}_{i}$ which is known to us. Since $y$ is the principal function, $\hat{y}_{i}$ is known and so then from (3.17)-(3.19), $\mu$ is known.

In the case that the principal function $y$ is identified with $e^{\left(\varphi-\varphi_{i} *\right) / \epsilon} v$, we similarly derive (3.17). But now

$$
\lambda=-\epsilon s_{i} / g_{i}
$$

approximately; and $\hat{w}_{i}$ is obtained from the normalized solution of $L^{*} y=0$, and is

$$
\hat{w}_{i}=\left(1 / f_{i}\right)\left|x_{i}-x_{i}\right|^{g_{i} / f_{i}^{\prime}} .
$$

For the minor function, the same development may be made, representing it as

$$
\bar{y}=\bar{\lambda}+\bar{\mu} \bar{w} \text {. }
$$


$\bar{\lambda}$ is computed as in (3.18), however since $\bar{y}$ is not known, we use a prior value of $\bar{y}$ (i.e., $\bar{y}$ from the previous iteration). The Eqs. (3.17)-(3.21) hold for the minor function with bars inserted as necessary. However, because $\bar{y}$ is the minor function, $\bar{y}_{i}$ is not known and so $\bar{\mu}$ is unknown as well. To determine $\bar{y}_{i}$, we must determine $\bar{\mu}$ and this will be done in the following paragraph.

3.4. Treatment at an Irregular Point. Our study of the principal function at neighboring points permits us to express this function as

$$
\lambda_{-}+\mu_{-} w_{-}
$$

to the left of an irregular point and as

$$
\lambda_{+}+\mu_{+} w_{+}
$$

to the right. Similarly, the minor functions to the left and to the right of an irregular point may be written as

$$
\bar{\lambda}_{-}+\bar{\mu}_{-} \bar{w}_{-}
$$

$$
\bar{\lambda}_{+}+\bar{\mu}_{+} \bar{w}_{+}
$$

respectively.

We may identify $\lambda_{+}+\bar{\lambda}_{+}+\bar{\mu}_{+} \bar{w}_{+}+\bar{\mu}_{+} \bar{w}_{+}$with (2.31)(a) as an approximation to the solution $y$, to one side of an irregular point. From Table 2.2 as $x_{i} \rightarrow$ $x_{i_{*}}$, the limiting values of $Y_{1}(x)$ and $Y_{2}(x)$ are known and approximate the normalized solutions (at $x_{i_{*}}$ ) which are $w_{+}$and $\bar{w}_{+}$in some order. Call $Y_{1}$ and $Y_{2}$ the functions $Y$ and $\bar{Y}$ as the case may be. Thus, by the continuity of $y$ at an irregular point, we have

(3.27) $\lambda_{-}+\bar{\lambda}_{-}+\mu_{-} Y_{-}(0)+\bar{\mu}_{-} \bar{Y}_{-}(0)=\lambda_{+}+\bar{\lambda}_{+}+\mu_{+} Y_{+}(0)+\bar{\mu}_{+} \bar{Y}_{+}(0)$.

Similarly, from (2.31)(b) and the continuity of $y^{\prime}$ at an irregular point, we have

$$
\mu_{-} Y_{-}^{\prime}(0)+\bar{\mu}_{-} \bar{Y}_{-}^{\prime}(0)=\mu_{+} Y_{+}^{\prime}(0)+\bar{\mu}_{+} \bar{Y}_{+}^{\prime}(0) .
$$

(3.27) and (3.28) form a system for the determination of $\mu_{+}$and $\bar{\mu}_{-}$. Except in the case where the determinant of this system vanishes (an analogue of the resonance phenomena), we may solve this system for $\bar{\mu}_{+}$and $\bar{\mu}_{-}$.

\section{Execution of the Algorithm.}

4.1. Comments and Limitations. (i) As we have remarked above, there must be at least two mesh points between each pair of irregular points.

(ii) The treatment of neighboring points and irregular points required division by $g$ (cf. (3.20)). Thus, as stated, the algorithm imposes the constraint that $g$ not vanish in the vicinity of an irregular point. Nevertheless, by ignoring the status of neighboring and irregular points and applying (3.4) and (3.5) to all points, an alternate algorithm is formally defined. Although we have not tested it, this alternate algorithm is probably reasonable in some cases. 
(iii) At an irregular point, the values of the right and left sided derivatives of $f$ (as well as the values of $g$ ) must not cause the vanishing of the determinant arising out of (3.27) and (3.28), for solving for $\bar{\mu}_{+}$and $\bar{\mu}_{-}$(i.e. the discrete analogue of the resonance phenomenon, already referred to, must be avoided).

(iv) If algorithmic resonance but not real resonance does occur, it can be eliminated by small changes in $f$ in the vicinity of irregular points. The effect of these changes is likely to result in small changes in the numerical results. For example, this is the case for those problems for which the maximum principle maintains.

4.2. An Algebraic Point of View. The algorithm yields in effect a linear system for the vectors $\hat{u}_{i}$ and $\hat{v}_{i}$. A special ordering of this linear system results in a coefficient matrix which in turn makes for an efficient solution procedure.

If $f_{i}<0$, we call $\hat{u}_{i}$ a rightward function, while we call it a leftward function if $f_{i}>0$. We use the reverse designations for $\hat{v}_{i}$. (This terminology devolves from the directional divided differences (3.2) and (3.3) as well as the wave-like behavior of the solution.)

Now, reorder the $\hat{u}_{i}$ and $\hat{v}_{i}$ so that all the rightward functions are contiguous and likewise the leftward functions. We also preserve the ordering of the subscript, $i$, within each of the two groups. The matrix $C$ of the resulting linear system has the following form.

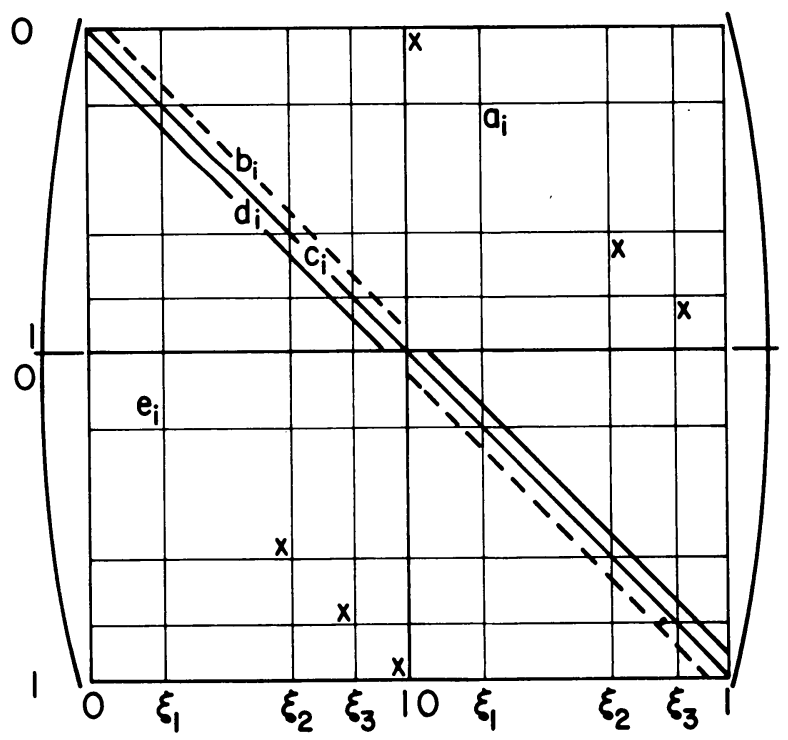

RIGHTWARD FUNCTION LEFTWARD FUNCTION

FIGURE 4.1. Schematic of the matrix of the linear system.

The $\xi_{i}$ correspond to the turning points 
Thus $C$ is a five diagonal matrix. The entries along the diagonals $a$ and $e$ are sparse and come from endpoints and the coupling in (3.27) and (3.28) (irregular points). The off-diagonal, $b$, has terms which are $O(\epsilon)$ corresponding to the rightward functions while the off-diagonal, $d$, has terms which are $O(\epsilon)$ corresponding to the leftward functions. (These $O(\epsilon)$ terms result respectively from the second divided difference terms in (3.4) or (3.5) as the case may be, and are schematized in Fig. 4.1 by the dashed segments.)

This form of $C$ suggests a relaxation procedure which consists of pairs of passes for solving the associated linear system.

(i) First Pass. Relax from left to right the equations corresponding to rightward functions.

(ii) Second Pass. 'Relax from right to left the equations corresponding to the leftward functions.

Coupling between the two passes occurs through the far off-diagonals $a$ and $e$. This method essentially results in a rapid movement of information flow by relaxation in the system, since the $O(\epsilon)$ coefficients of $C$ are arranged so that they do not slow down this flow. Because the nonzero entries in $C$ are of widely different magnitude, a relaxation step produces widely varying corrections in different variables. We found it necessary to smooth out this instability by use of the method of false position. In particular, let $\zeta=\left(\hat{u}_{1}, \hat{u}_{2}, \cdots, \hat{u}_{n}, \hat{v}_{1}, \cdots, \hat{v}_{n}\right)$ and let the result of a relaxation without smoothing be represented by $F\left(\zeta^{m-1}\right)$. Including the smoothing we have

$$
\zeta_{i}^{m+1}=\frac{F_{i}\left(\zeta^{m-1}\right) \zeta_{i}^{m}-F_{i}\left(\zeta^{m}\right) \zeta_{i}^{m-1}}{\zeta_{i}^{m}-\zeta_{i}^{m-1}+F_{i}\left(\zeta^{m-1}\right)-F_{i}\left(\zeta^{m}\right)}
$$

For small $\epsilon$, the algorithm converges in a few iterations ( 4 or 5 for the cases of interest); the fewer, the smaller $\epsilon$.

5. Numerical Results. In subsection 5.1, we characterize the errors arising through use of our algorithm corresponding to various cases for the equation $\epsilon y^{\prime \prime}+$ $a x y^{\prime}+b y=0$. In subsection 5.2, we give a compendium of examples for linear equations with a variety of variable coefficients. We also include some of the results of C. E. Pearson [6] for comparison.

5.1. Solution of the Boundary Value Problem $\epsilon y^{\prime \prime}+a x y^{\prime}+b y=0$ with $y(-1)=1, y(1)=2$. We take $\epsilon=10^{-7}$. Two typical cases are

(i) $a=-1, b<0$,

(ii) $a=1, b=-1.0001(\sim-1)$.

In case (i), the algorithm gives zero at all interior mesh points if $b$ is not a (machine) integer. This corresponds to an error of less than $10^{-8}$.

In case (ii), the exact solution is linear to within $10^{-4}$ on each side of the turning point, where it vanishes. Fig. 5.1 displays the approximate solution. In Fig. 5.2, we 
plot the maximum error versus $\zeta$, the mesh increment $\Delta x$. The linearity of the plot in Fig. 5.2 is not surprising since all of the discretization procedures in the various aspects of the algorithm were of the first order in $\Delta x$.

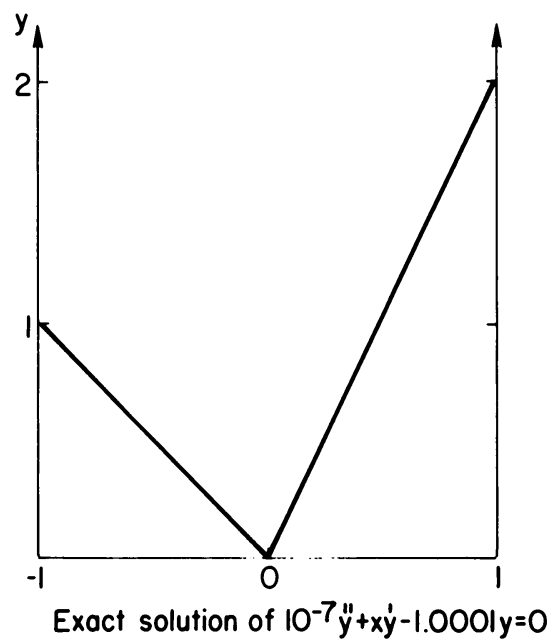

FIgURE 5.1. Approximate solution in case (ii)

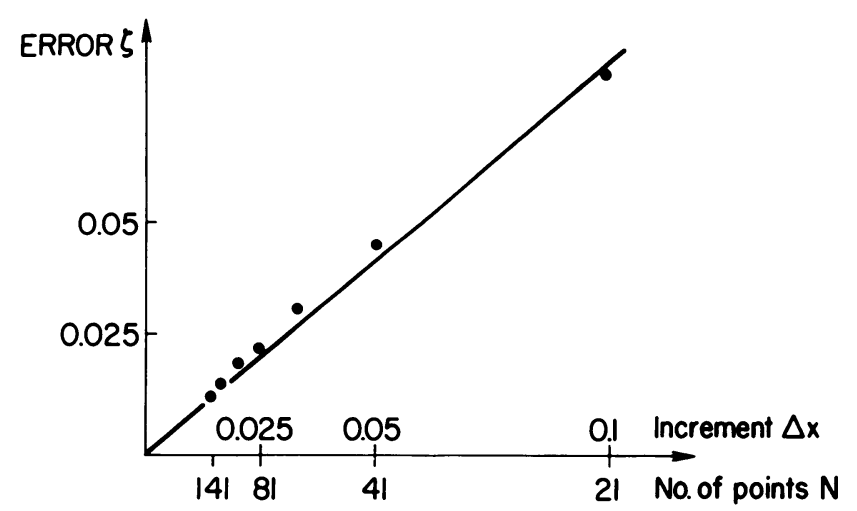

Numerical error for $10^{-7} \ddot{y}+x y-1.0001 y=0$

FIGURE 5.2. Maximum error $\Delta x$ in case (ii)

5.2. Variable Coefficient Cases. In this subsection we present a sequence of figures exhibiting the coefficient $f(x)$ of the term $y^{\prime}$, and the corresponding results.

Pearson [6] has studied some of these cases by a refinement method. We get exactly the same results as he in the cases: 5.3, 5.5, 5.6, 5.7; and close to his results in cases: $5.4,5.8,5.9,5.10,5.11,5.12$. We compare also our result to an exact solution in cases: $5.14,5.15,5.16$. In all these cases, the difference goes to zero with the mesh increment and, when the maximum principle is satisfied, is less than the mesh increment. 
TURNING POINTS IN STIFF BOUNDARY VALUE PROBLEMS

1031
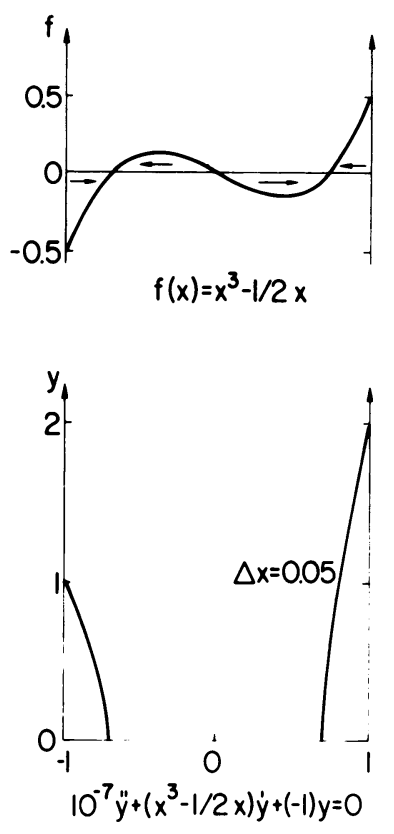

FigURE 5.3
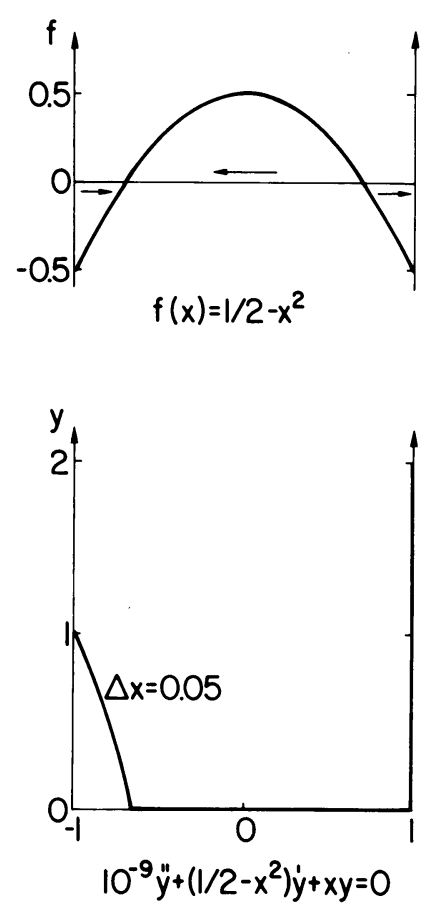

FIGURE 5.5
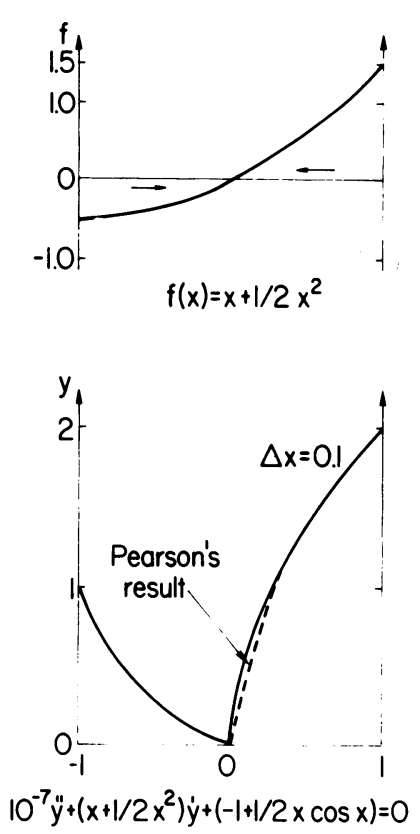

FIGURE 5.4
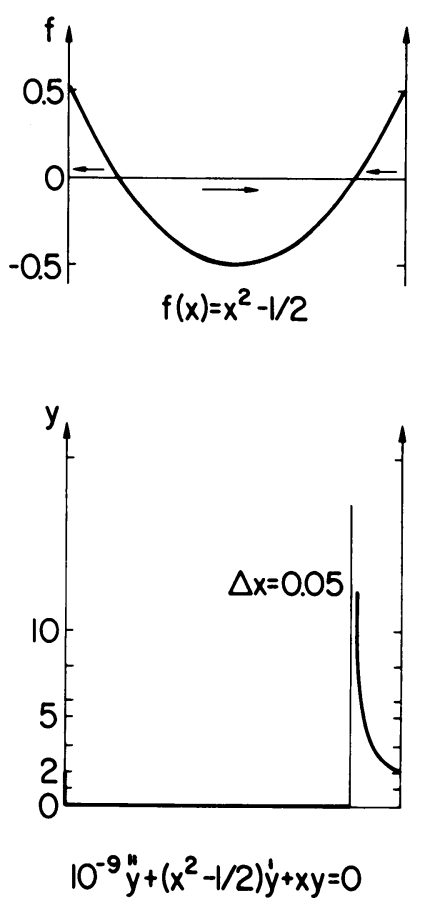

FIGURE 5.6 

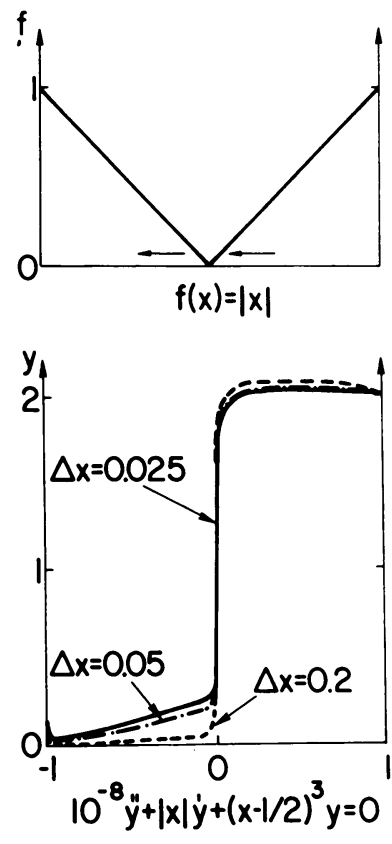

FIGURE 5.7
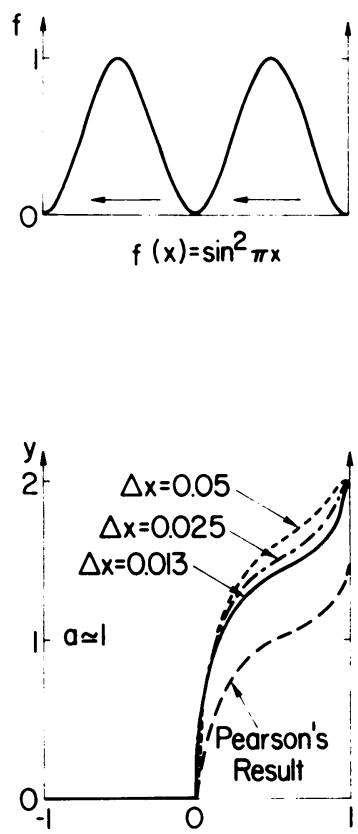

$10^{-7} \ddot{y}+(\sin \pi x)^{2} \dot{y}+(x-a) y=0$

FIGURE 5.9

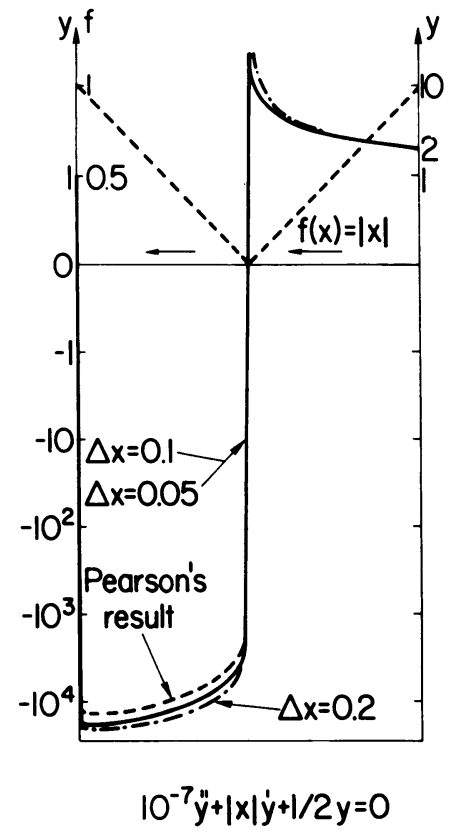

FiguRE 5.8
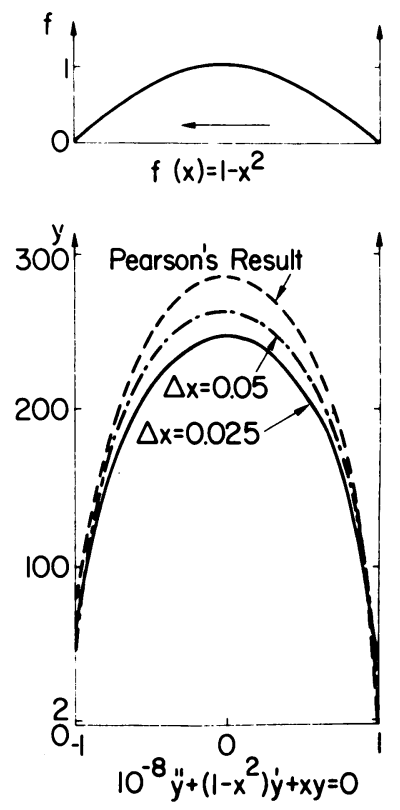

FIGURE 5.10 


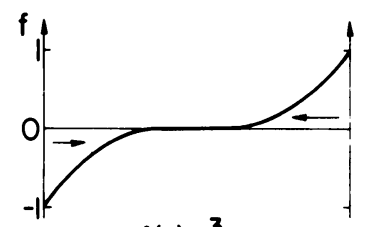

$f(x)=x^{3}$

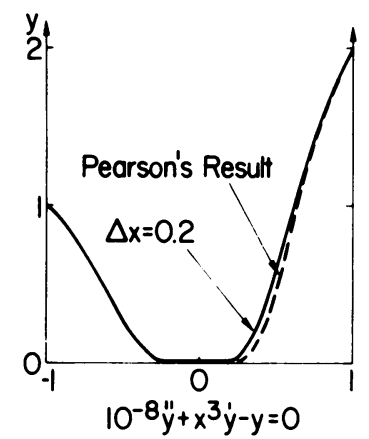

Vibration case : $f^{\prime}(0)=0$

Figure 5.11

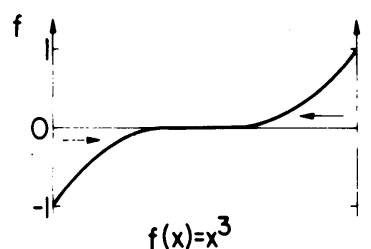

3 cycles of oscillations with amplitude $10^{20}$

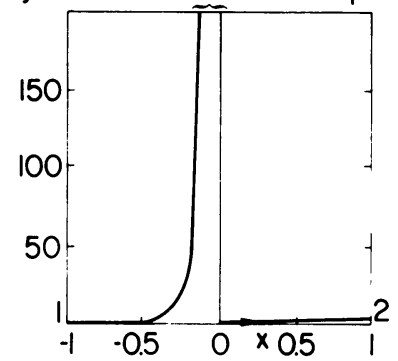

Pearson's Result for $10^{-8} \ddot{y}+x^{3} y-x y=0$

In this case, our method is unstable

FIGURE 5.12

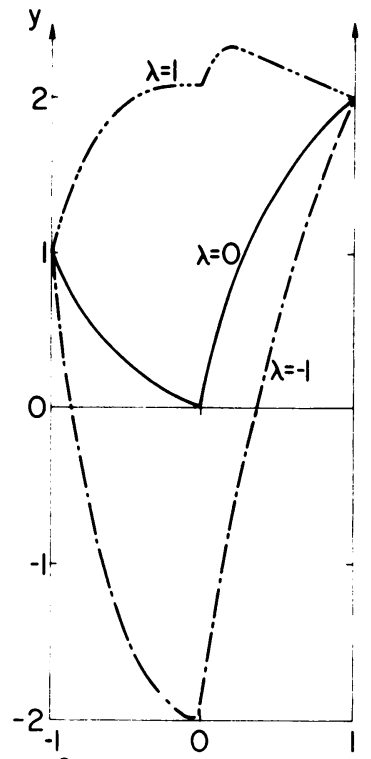

$10^{-7} \ddot{y}+\left(x+1 / 2 x^{2}\right) \dot{y}+(-1+1 / 2 x \cos x) y=\lambda(-3+\sin (x+\cos x))$

FIGURE 5.13

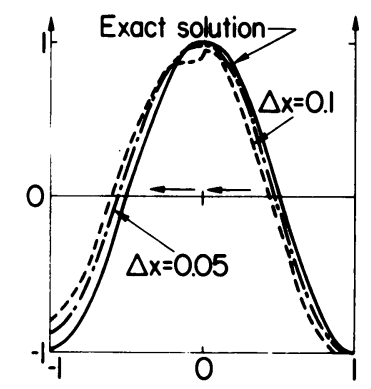

$10^{-7} \ddot{y}+|x| \dot{y}-y=-\left(1+\pi^{2} 10^{-7}\right) \cos \pi x-\pi|x| \sin \pi x$

Exact solution: $y=\cos \pi x$

Elliptic equation

FIGURE 5.14 

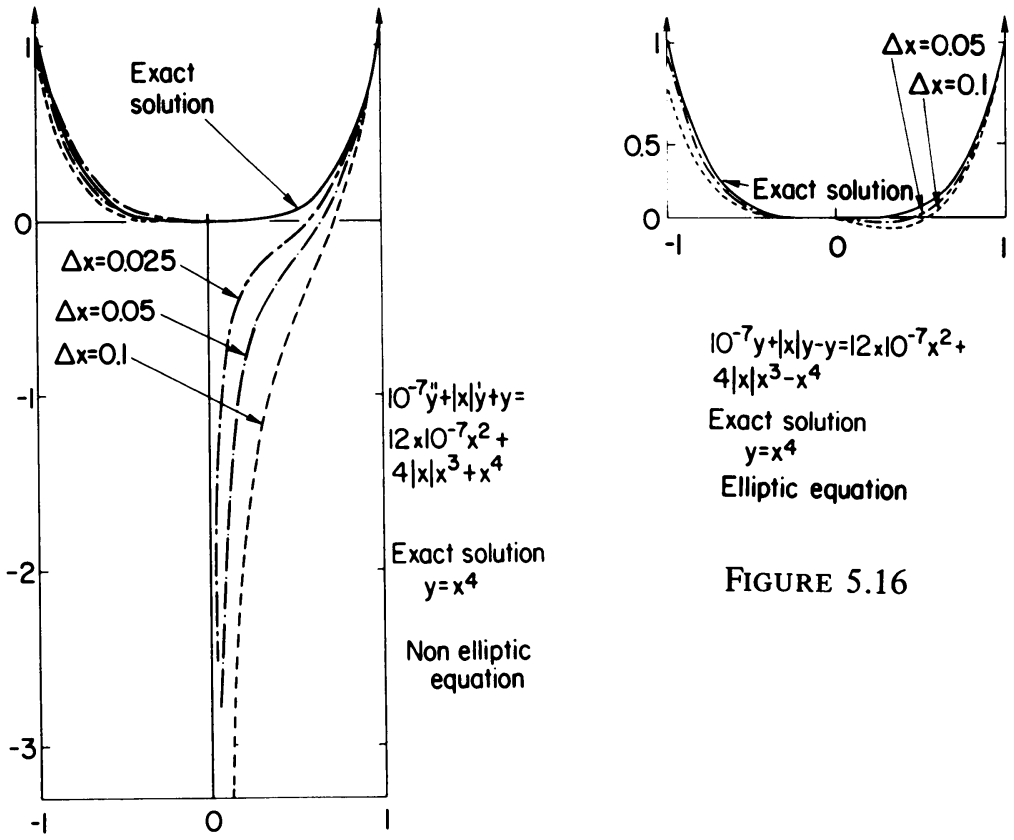

$10^{-7} y+|x| y-y=12 \times 10^{-7} x^{2}+$ $4|x| x^{3}-x^{4}$

Exact solution $y=x^{4}$

Elliptic equation

Figure 5.16

FIGURE 5.15

IBM Thomas J. Watson Research Center Yorktown Heights; New York 10598

1. F. W. DORR, "An example of ill-conditioning in the numerical solution of singular perturbation problems," Math. Comp., v. 25, 1971, pp. 271-283.

2. F. W. DORR, S. V. PARTER \& L. F. SHAMPINE, "Application of the maximum principle to singular perturbation problems," SIAM Rev., v. 15, 1972, pp. 43-88.

3. A. M. IL'IN, "A difference scheme for a differential equation with a small parameter multiplying the highest derivative," Mat. Zametki, v. 6, 1969, pp. 237-248=Math. Notes, v. 6, 1969, pp. 596-602. MR 41 \#4823.

4. W. L. MIRANKER, "Numerical methods of boundary layer type for stiff systems of ordinary differential equations," Computing, v. 11, 1973, pp. 221-234.

5. R. E. O'MALLEY, Introduction to Singular Perturbation, Lecture Notes at Edinburgh, Autumn, 1971.

6. C. E. PEARSON, "On a differential equation of boundary layer type," J. Mathematical Phys., v. 47, 1968, pp. 134-154. MR 37 \#3773. 